\title{
Effect of the temperature dependence of gas absorption in climate feedback
}

\author{
Yi Huang ${ }^{1}$ and V. Ramaswamy ${ }^{2}$ \\ Received 12 April 2006; revised 25 November 2006; accepted 13 December 2006; published 3 April 2007.
}

[1] In the context of climate feedback associated with temperature change, there exist two potential mechanisms that affect the outgoing longwave radiation (OLR) and the downward longwave radiation (DLR). One is the "Planck" effect that determines the blackbody thermal emission at a considered temperature. The other is the "absorptivity" effect, in which a temperature change causes a change in gas absorptivities and thus influences the longwave radiative transfer. By using the line-by-line computed radiative Jacobians, which quantify the sensitivity of the radiative fluxes to a perturbation in the atmospheric temperature, the absorptivity effect is separated from the Planck effect. The absorptivity effect is further partitioned into components, with each one having a distinct physical meaning. It is demonstrated that the absorptivity-induced changes in the longwave radiation are individually significant even though the net effect is largely one of cancellation. As a consequence, the Planck effect dominates the overall OLR and DLR sensitivities to temperature change. The absorptivity effect tends to counteract the Planck effect. This tendency is particularly significant for the DLR and is more prominent for a warmer climate, with the result being a reduction in the surface warming.

Citation: Huang, Y., and V. Ramaswamy (2007), Effect of the temperature dependence of gas absorption in climate feedback, J. Geophys. Res., 112, D07101, doi:10.1029/2006JD007398.

\section{Introduction}

[2] A change in the Earth's surface temperature is necessarily accompanied by a change in the vertical profile of the atmospheric temperature [Manabe and Wetherald, 1967]. The change in atmospheric temperature further perturbs the Earth's radiative energy budget, generating a feedback onto the initial surface temperature change. For convenience, we will term this process as "temperature feedback," which constitutes one aspect of the climate feedback. The temperature feedback has been shown to be one of the most important mechanisms [Zhang et al., 1994; Stocker et al., 2001] that damp the climate warming initially induced by external radiative perturbations, e.g., the anthropogenic increase of carbon dioxide. Atmospheric temperature directly influences the thermal emission according to Planck's Law [Goody and Yung, 1989]. In addition, the absorptivities of the various gaseous components in the atmosphere, being temperature-dependent, influence the transmission and emission of radiation energy following Kirchhoff's Law. The temperature dependence of gas absorption, arising from the dependence of line strength and line width on temperature [Goody and Yung, 1989], has long been recognized as a necessary component in radiative transfer

\footnotetext{
${ }^{1}$ Program in Atmospheric and Oceanic Sciences, Princeton University, Princeton, New Jersey, USA.

${ }^{2}$ Geophysical Fluid Dynamics Laboratory, NOAA, Princeton, New Jersey, USA.

Copyright 2007 by the American Geophysical Union. 0148-0227/07/2006JD007398
}

computations, especially in radiatively forced climate change problems. However, the "gas absorptivity" effect on temperature feedback has not yet been adequately highlighted and distinguished from the "Planck" emission effect. On the one hand, from earlier model-based climate sensitivity studies [e.g., Augustsson and Ramanathan, 1977] it emerges that the neglect of the temperature dependence of gas absorption results in an overestimation of the climate sensitivity, although reasons for this have not been explicitly given. On the other hand, temperature feedback is often simply designated as the "Planck effect" in the literature; this actually masks the presence of the temperature-dependent gas absorptivity effect. So, it is of great theoretical interest to quantify the effect due to the temperature dependency of gas absorptivities in the context of climate feedback, to determine how it contributes to the temperature feedback, and to compare it with the betterdefined Planck effect. Moreover, it is also of practical concern to understand whether and how the temperature feedback may change under a changing (e.g., a warmer) climate due to this effect.

[3] Because of the substantial variation of temperature and gas concentrations with height, and the differing temperature dependence of water vapor continuum absorption and lines, the investigation of the above questions requires the use of a spectrally and vertically resolved model. Recent works, such as Soden and Held [2006] and Huang et al. [2007], have shown that the temperature and water vapor feedbacks can be evaluated using radiative Jacobians. These Jacobians represent the sensitivities of the radiative fluxes to water vapor and temperature perturbations, as a function of spectral interval and altitude. This paper extends these 
previous studies to a diagnosis of the Planck and gas absorptivity effects in the Jacobian framework. Our analysis focuses on the Outgoing Longwave Radiation (OLR) at the Top of the Atmosphere (TOA) and Downward Longwave Radiation (DLR) at the surface under clear skies. We employ the Line-by-line (LBL) radiative transfer model of the Geophysical Fluid Dynamical Laboratory (GFDL) [Fels and Schwarzkopf, 1981; Schwarzkopf and Ramaswamy, 1999] for the computations.

\section{Method}

\subsection{Longwave Radiative Transfer Formulation}

[4] Assuming the Earth's atmosphere to be plane-parallel and neglecting scattering, [Goody and Yung, 1989], the monochromatic outgoing longwave radiance at TOA and the downward longwave radiance at surface can be expressed, in discrete form, as:

$$
\begin{aligned}
I_{\nu}^{+}(0, \mu)= & B_{\nu}\left(T_{s}\right) \bullet \operatorname{Tr}_{\nu}\left(\tau_{s}, 0 ; \mu\right) \\
& +\sum_{i=1}^{N+1} B_{\nu}\left(T_{i}\right) \bullet\left[\operatorname{Tr}_{\nu}\left(\tau_{i}, 0 ; \mu\right)-\operatorname{Tr}_{\nu}\left(\tau_{i+1}, 0 ; \mu\right)\right] \\
I_{\nu}^{-}\left(\tau_{s}, \mu\right) & =\sum_{i=1}^{N+1} B_{\nu}\left(T_{i}\right) \bullet\left[\operatorname{Tr}_{\nu}\left(\tau_{i+1}, \tau_{s} ; \mu\right)-\operatorname{Tr}_{\nu}\left(\tau_{i}, \tau_{s} ; \mu\right)\right]
\end{aligned}
$$

[5] Here, $B_{\nu}(T)$ is the thermal emission at wave number $\nu$ and temperature $T$ according to Planck's Law (the subscript $s$ denotes surface); $\operatorname{Tr}_{\nu}(\tau, 0 ; \mu)$ is the transmission function from the level where the optical depth is $\tau$ to the TOA where it is 0 , and $\mu$ is the cosine of the zenith angle. $\operatorname{Tr}_{\nu}(\tau, 0 ; \mu)=$ $e^{-\tau / \mu} ; \tau$ is the integral of the product of absorption coefficient and absorber path length from TOA to the altitude level considered. The absorptivity, $A_{\nu}$, is equal to $1-T r_{\nu}$. $N$ is the number of discretized atmospheric layers, with the first layer being at the top, and the $N$ th next to the surface.

[6] OLR is the outcome of the angular and spectral integration of $I_{\nu}^{+}(0, \mu)$; and DLR, the corresponding integration for $I_{\nu}^{-}\left(\tau_{s}, \mu\right)$.

$$
\begin{aligned}
& O L R=2 \pi \int_{0}^{1} d \mu \int_{\nu 1}^{\nu 2} \mu I_{\nu}^{+}(0, \mu) d \nu \\
& D L R=2 \pi \int_{-1}^{0} d \mu \int_{\nu 1}^{\nu 2} \mu I_{\nu}^{-}\left(\tau_{s}, \mu\right) d \nu
\end{aligned}
$$

$v 1$ and $v 2$ are the lower and upper limits of the longwave spectral range; here, we use 0 and $2500 \mathrm{~cm}^{-1}$, respectively. In our LBL model, a four-point Gaussian quadrature method [Fels and Schwarzkopf, 1981] is used to perform the angular integration to obtain the OLR and DLR.

[7] The temperature dependence of the gas absorption (emission) arises owing to the functional dependence of the line strengths and line widths on temperature [Goody and Yung, 1989]. The line strengths are related to the population distributions of the molecular energy states, which in turn depend on temperature. The broadening of the spectral lines (line width), caused by either collisional (Lorentz) broadening or Doppler broadening, is also a function of temperature. The temperature dependence of line absorption varies from line to line. On the other hand, water vapor continuum absorption, though still not fully understood on a fundamental basis, is known to decrease with temperature according to available but as yet limited measurements (at least in the window region) [Comier et al., 2005]; it is, however, reasonably well represented by an empirical formula [Clough et al., 1989]. The exact mechanisms that cause these two types of absorption to be temperature-dependent are different and beyond the scope of this paper, though it is worth pointing out that the empirical formulation of the continuum absorption is inevitably a source of some uncertainty.

[8] As an example, the temperature dependence of gas (water vapor only) absorptivity is demonstrated in Figure 1, using the GFDL LBL model. The calculations are based upon the HITRAN spectral line data [Rothman et al., 2003] and CKD2.4 water vapor continuum absorption formulation [Tobin et al., 1999]. Temperature and water vapor follow the standard tropical profiles [McClatchey et al., 1972]. There are $4520-\mathrm{hPa}$ thick layers between $1000 \mathrm{hPa}$ and $100 \mathrm{hPa}$, and 15 layers between $100 \mathrm{hPa}$ and $10 \mathrm{hPa}$, with the layer thickness decreasing exponentially with height. In this LBL calculation, $\mathrm{a}+1 \mathrm{~K}$ temperature perturbation results in a decrease of narrow band $\left(1 \mathrm{~cm}^{-1}\right)$ layer absorptivity in the atmospheric window and the centers of absorption bands, but results in an absorptivity increase for those lines which are off the band center.

\subsection{Partitioning of the Planck and Gas Absorptivity Effects}

[9] The question of interest here is how the Planck and absorptivity effects, respectively, contribute to the spectral OLR (DLR) change when there is a change in the atmospheric temperature. As in the work by Huang et al. [2007], contributions from each of the two factors can be separated, on the basis of a Taylor expansion. For example, a $1 \mathrm{~K}$ increase of the temperature of a layer $j$ will cause the following terms to change: $B_{\nu}\left(T_{j}\right)$, because of the Planck effect; $\operatorname{Tr}\left(\tau_{s}, 0 ; \mu\right), \operatorname{Tr}\left(\tau_{i}, 0 ; \mu\right)(i>j)$ in the case of OLR, and $\operatorname{Tr}\left(\tau_{i}, \tau_{s} ; \mu\right)(i<j)$ in the case of DLR, because of the temperature-dependent absorptivity effect. By neglecting the higher-order residual terms, the change of $I_{\nu}^{+/-}$can be written as:

$$
\begin{aligned}
& \Delta I_{\nu}^{+}(0, \mu)=\Delta B_{\nu}\left(T_{j}\right) \bullet\left[\operatorname{Tr}_{\nu}\left(\tau_{j}, 0 ; \mu\right)-\operatorname{Tr}_{\nu}\left(\tau_{j+1}, 0 ; \mu\right)\right] \\
& +B_{\nu}\left(T_{j}\right) \bullet\left[\Delta \operatorname{Tr}_{\nu}\left(\tau_{j}, 0 ; \mu\right)-\Delta \operatorname{Tr}_{\nu}\left(\tau_{j+1}, 0 ; \mu\right)\right] \\
& +\sum_{i=j+1}^{N} B_{\nu}\left(T_{i}\right) \bullet\left[\Delta \operatorname{Tr}_{\nu}\left(\tau_{i}, 0 ; \mu\right)-\Delta \operatorname{Tr}_{\nu}\left(\tau_{i+1}, 0 ; \mu\right)\right] \\
& +B_{\nu}\left(T_{S}\right) \bullet \Delta \operatorname{Tr}_{\nu}\left(\tau_{s}, 0 ; \mu\right) \\
& \Delta I_{\nu}^{-}\left(\tau_{s}, \mu\right)=\Delta B_{\nu}\left(T_{j}\right) \bullet\left[\operatorname{Tr}_{\nu}\left(\tau_{j+1}, \tau_{s} ; \mu\right)-\operatorname{Tr}_{\nu}\left(\tau_{j}, \tau_{s} ; \mu\right)\right] \\
& +B_{\nu}\left(T_{j}\right) \bullet\left[\Delta \operatorname{Tr}_{\nu}\left(\tau_{j+1}, \tau_{s} ; \mu\right)-\Delta \operatorname{Tr}_{\nu}\left(\tau_{j}, \tau_{s} ; \mu\right)\right] \\
& +\sum_{i=j-1}^{1} B_{\nu}\left(T_{i}\right) \bullet\left[\Delta \operatorname{Tr}_{\nu}\left(\tau_{i+1}, \tau_{s} ; \mu\right)-\Delta \operatorname{Tr}_{\nu}\left(\tau_{i}, \tau_{s} ; \mu\right)\right]
\end{aligned}
$$


a) Absorptivity

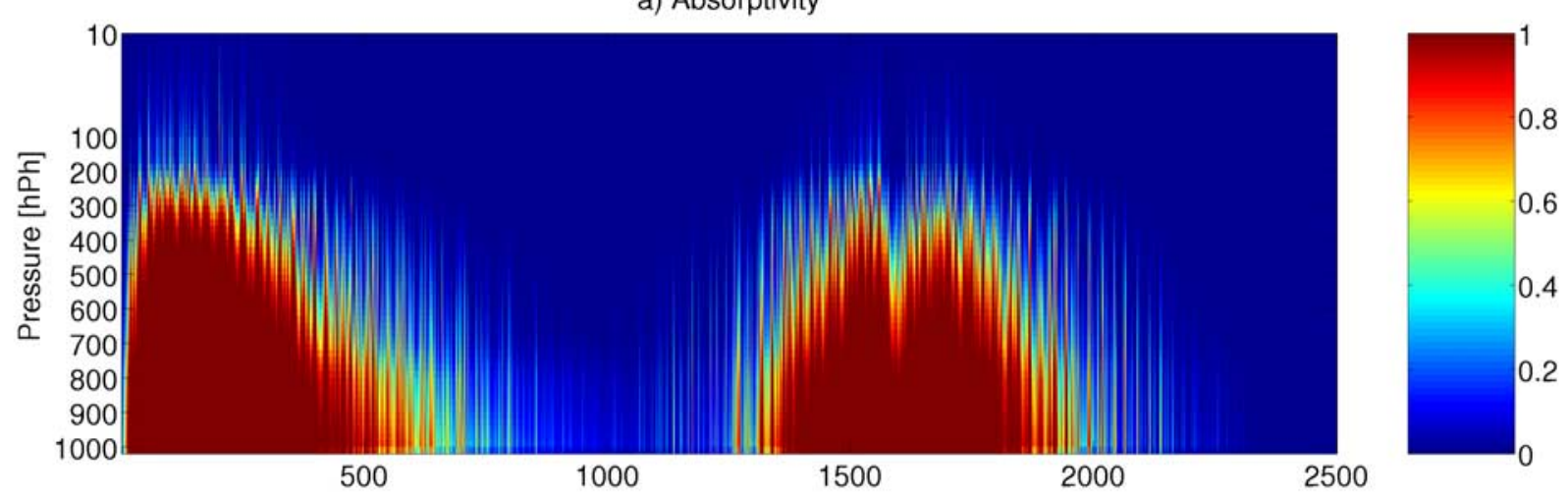

b) Absorptivity change

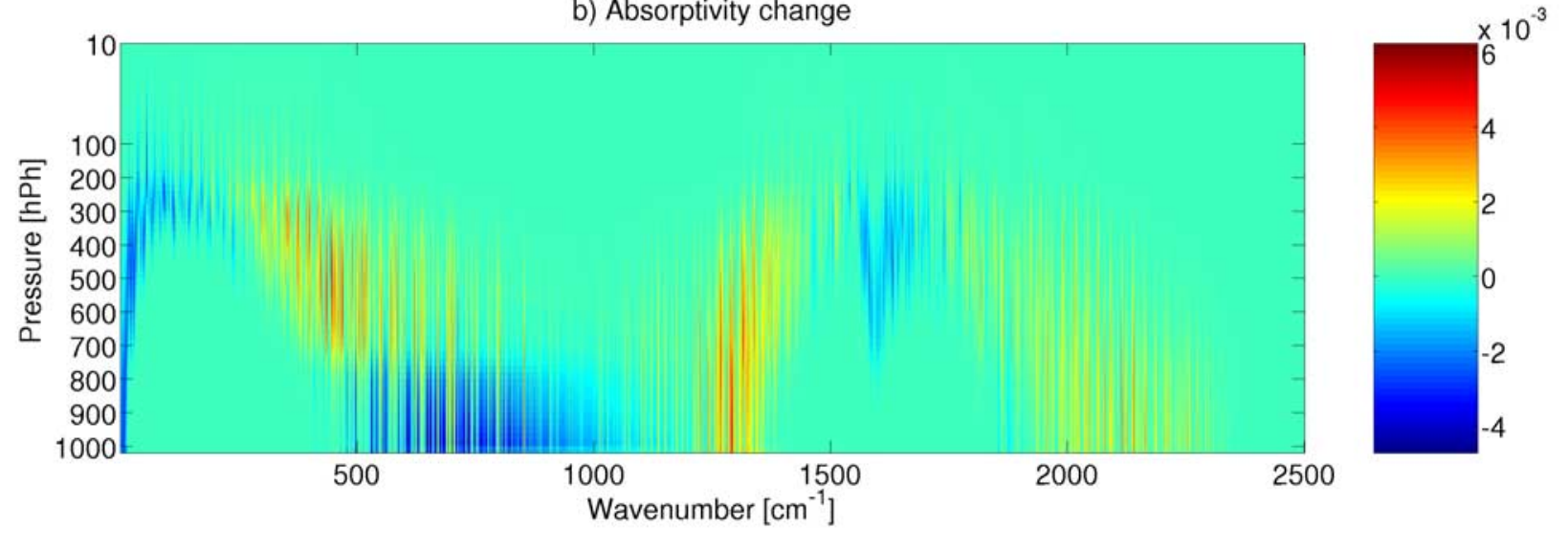

Figure 1. Layer absorptivity of water vapor in a tropical atmosphere: (a) layer absorptivity at each $1 \mathrm{~cm}^{-1}$ spectral interval and (b) its change due to $\mathrm{a}+1 \mathrm{~K}$ temperature perturbation.

Here $\Delta X$ denotes the change in variable $X$. Term (5a) corresponds to the change in the thermal emission by the perturbed layer due to the Planck effect; the other three terms all result from the temperature-dependent absorptivity effect, but have different origins as follows: Term (5b) is the change in the outgoing radiation resulting from the perturbed layer; term $(5 \mathrm{c})$ results from the change in transmission functions from the atmospheric layers below the perturbed layer to TOA; and term (5d) results from the change in transmission functions from the surface to TOA. Analogously, in equation (6), the three terms correspond, respectively, to the Planck effect on DLR (term (6a)), the absorptivity effect on downward emission from the perturbed layer (term (6b)), and the absorptivity effect on downward emission from the layers above (term (6c)).

[10] A central purpose of this paper is to illustrate how the contribution by each of the above terms is distributed with respect to spectral frequency and altitude of temperature perturbation. Using the GFDL LBL model, each of these four terms is evaluated. Besides the features introduced in section 2.1, this model takes temperature, water vapor and ozone profiles from McClatchey et al. [1972], and wellmixed greenhouse gas $\left(\mathrm{CO}_{2}, \mathrm{CH}_{4}, \mathrm{~N}_{2} \mathrm{O}, \mathrm{CFC}-11, \mathrm{CFC}-12\right.$, CFC-113 and HCFC-22) concentrations as appropriate to the year 2000. The model used in the subsequent text has
25 vertical levels (The computation results are not affected by this lower resolution [Huang et al., 2007]).

\section{Results}

\subsection{OLR}

[11] We define the spectral Jacobians of OLR as the change in the outgoing irradiance flux in every $1 \mathrm{~cm}^{-1}$ spectral interval resulting from a $+1 \mathrm{~K}$ temperature perturbation of a 50-mbar thick layer at a specific height in the atmosphere. They are calculated using the standard tropical profiles. These Jacobians are further decomposed in Figure 2 into four parts (Figures $2 a-2 d$ ), illustrating the four terms on the RHS of equation (5) in order. The sum of the four terms (not shown), is almost identical to the total OLR change when both Plank and absorptivity effects are considered simultaneously [Huang et al., 2007, Figure 2b], which confirms the validity of the separation in equation (5).

[12] As illustrated in Figure 2a, the Planck effect gives rise to an unambiguous increase of OLR, with the maximum contribution in the $\mathrm{CO}_{2} 15$ micron $\left(667 \mathrm{~cm}^{-1}\right)$ band. Figure 2a illustrates the origin of the strong Planck damping effect in a warmer climate. It correlates very well with the atmospheric contribution to OLR [cf. Clough et al., 1992, Plate 4]). In the water vapor rotation and vibration-rotation 

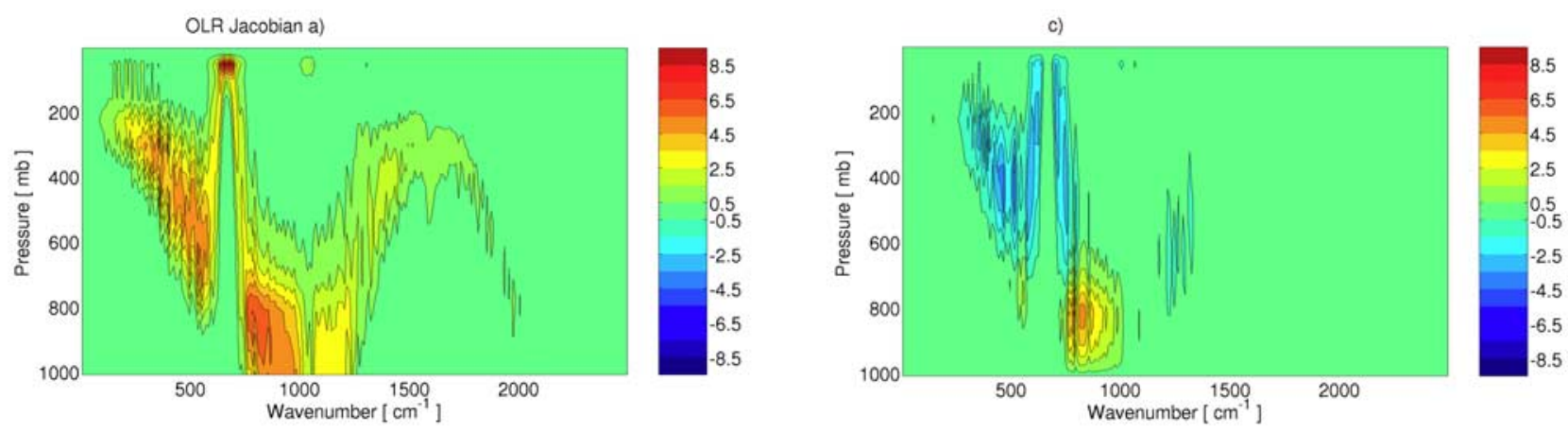

b)

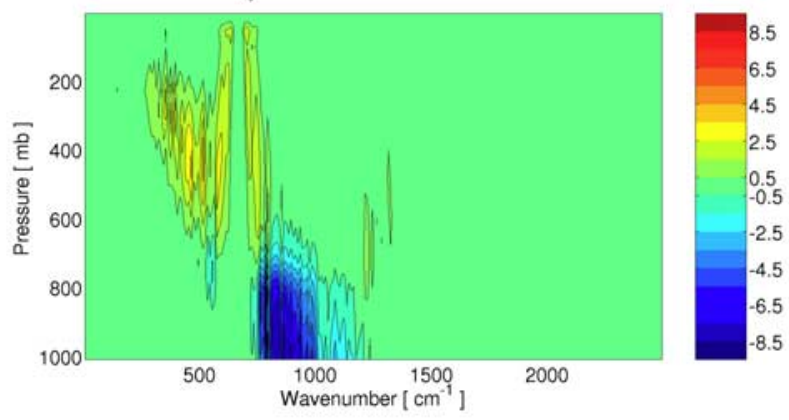

d)

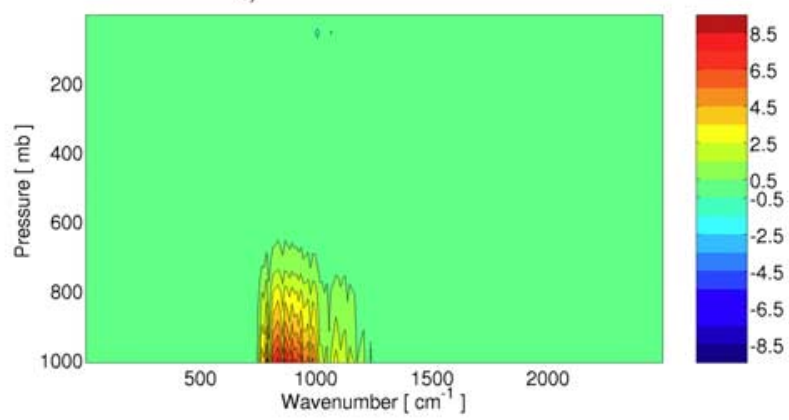

Figure 2. Spectral Jacobians of OLR separated into four parts, corresponding to the four terms in the right-hand side of equation (5), respectively: (a) Planck effect on the outgoing radiation from the perturbed layer, (b) gas absorptivity effect on the outgoing radiation from the perturbed layer, (c) gas absorptivity effect on the outgoing radiation from the layers below, and (d) gas absorptivity effect on the outgoing radiation from the surface. $\mathrm{A}+1 \mathrm{~K}$ temperature perturbation, applied successively in $50 \mathrm{hPa}$ thick layers, is considered. The results are contoured in wave number-pressure space, with an interval of $1 \mathrm{e}-4 \mathrm{~W} \mathrm{~m}^{-2} / \mathrm{cm}^{-1}$.

bands, most of the outgoing radiation, as well as most of the damping under a warming perturbation, arises from the middle and upper troposphere; in the carbon dioxide 15 micron and the ozone 9.6 micron $\left(1042 \mathrm{~cm}^{-1}\right)$ bands, it is mostly from the stratosphere; and in the atmospheric window region $\left(\sim 8-12\right.$ micron, i.e., $\left.830-1250 \mathrm{~cm}^{-1}\right)$, it is mostly from the lower troposphere.

[13] Figure $2 \mathrm{~b}$ illustrates the contribution from the absorptivity effect term (5b), namely, the change of OLR due to the change in the temperature-dependent emissivity (absorptivity) of the perturbed layer only. Below $830 \mathrm{~cm}^{-1}$ or above $1250 \mathrm{~cm}^{-1}$, the layer emissivity increases (Figure 1b), owing to the unsaturated weaker lines. This gives rise to a larger thermal emission from the perturbed layer and thus increases OLR. In the window region, on the contrary, the continuum absorption coefficient decreases (Figure 1b) and this results in less outgoing radiation.

[14] Figure 2c reflects term (5c), namely, the change in the outgoing radiation emitted by the layers below the perturbed layer. Figure $2 \mathrm{c}$ is almost the negative of Figure $2 \mathrm{~b}$. In the window region, where transmission increases because of the negative temperature dependence of continuum absorption, more emission from the layers below reaches TOA; outside of the window region, more outgoing radiation is trapped.

[15] The change in the outgoing radiation from the surface, Figure 2d, occurs only in the window region, and mainly when the temperature in the lower troposphere is perturbed. Because the absorption in the other spectral regions is already saturated, the temperature-influenced absorptivity change has no effect there.

[16] Figure 3 illustrates the integrated Jacobians, i.e., the change of the integrated irradiance flux over the whole longwave spectrum of OLR due to each of the four terms. Comparing Figure $2 \mathrm{a}$ of this paper to Figure $2 \mathrm{~b}$ of Huang et al. [2007], or the Planck effect curve to the total OLR change curve in Figure 3, it is apparent that the Planck effect largely resembles the total OLR change. This is because terms $(5 b)-(5 c)$, though individually significant, cancel, thus making the overall absorptivity influence small.

[17] It is noted that the OLR sensitivity to atmospheric temperatures does differ from the Planck effect, overshooting slightly in the lower troposphere while undershooting slightly in the upper and middle troposphere (Figure 3). To understand these features physically, it is helpful to resort to a conceptual picture. Let us consider the sum of terms $(5 \mathrm{c})$ and $(5 \mathrm{~d})$ to be the change of outgoing radiation from a hypothetical layer. This layer has an optical path length to TOA that is approximately the same as layer $j$, and has an equivalent emitting temperature, $T_{j}^{\prime}$. If this temperature was the same as $T_{j}$ (e.g., an isothermal atmosphere), the total absorptivity effect would be identically zero because term (5b) cancels the sum of terms $(5 c)$ and $(5 d)$. However, since the tropospheric thermal structure is such that the temperature decreases with height, $T_{j}^{\prime}>T_{j}$. Hence, in the spectral regions where the temperature perturbation $(+1 \mathrm{~K})$ 


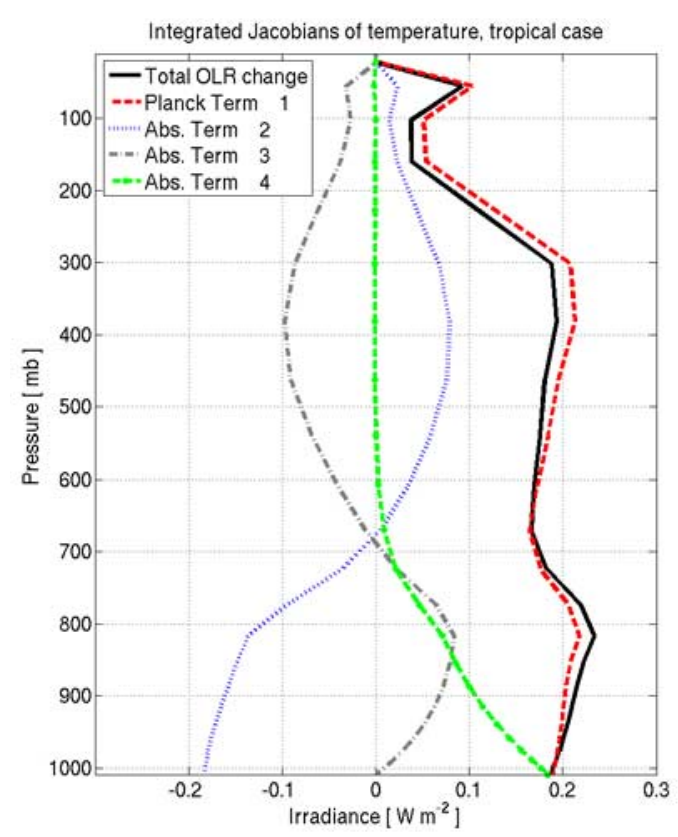

Figure 3. Integrated Jacobians $\left(\mathrm{W} \mathrm{m}^{-2}\right)$ of OLR, i.e., spectral integral of the Jacobians in Figure 2. The total outgoing irradiance flux change (solid curve) is separated into four parts, as in equation (5).

increases (decreases) the absorptivity of layer $j$, the absorptivity effect contributes negatively (positively) to the OLR change. It then becomes clear that the overshooting in the lower troposphere is due to the continuum absorption in the window region which decreases with temperature, while the undershooting in the middle and upper troposphere is due to those lines whose absorption strength increases with temperature (see Figure 4).

[18] From Table 1, for a vertically uniform temperature change of $+1 \mathrm{~K}$ in the tropics, the spectrally integrated OLR flux change is $3.40 \mathrm{~W} \mathrm{~m}^{-2}$, to which the Planck effect contributes $3.52 \mathrm{~W} \mathrm{~m}^{-2}$ and the absorptivity effect $-0.11 \mathrm{~W} \mathrm{~m} \mathrm{~m}^{-2}$. As illustrated in Figure 4, the overall absorptivity effect consists of offsetting contributions from different spectral regions. The spectral regions with a

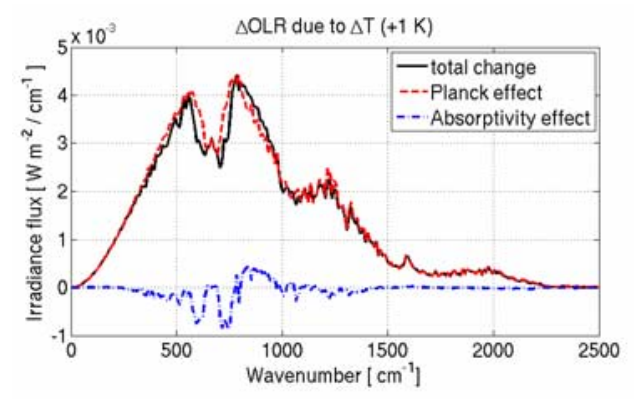

Figure 4. OLR flux ( $\mathrm{W} \mathrm{m}-2 / \mathrm{cm}^{-1}$ ) change due to $+1 \mathrm{~K}$ temperature perturbation. The result is vertically integrated from the spectral Jacobians. The total OLR change is separated into the contributions by the Planck and gas absorptivity effects.
Table 1. Column Sum of the Irradiance Jacobians of OLR ${ }^{\mathrm{a}}$

\begin{tabular}{llll}
\hline & Tropics & MLS & MLW \\
\hline Total OLR change & 3.40 & 3.04 & 2.11 \\
Planck effect & 3.52 & 3.19 & 2.25 \\
Absorptivity effect & -0.11 & -0.15 & -0.15 \\
$\begin{array}{l}\text { Absorptivity effect on outgoing } \\
\text { radiation from the surface }\end{array}$ & 0.56 & 0.39 & 0.08 \\
\hline
\end{tabular}

${ }^{\mathrm{a}}$ Unit is $\mathrm{W} \mathrm{m}^{-2}$. Three standard atmospheres are considered: tropics, midlatitude summer (MLS), and midlatitude winter (MLW). The values are vertically integrated from the Jacobians illustrated in Figure 3 and represent the OLR change when the temperature of the whole atmospheric column changes uniformly by $1 \mathrm{~K}$.

negative contribution (owing to the lines with positive temperature dependence) override the regions with a positive contribution (owing to the continuum absorption and the rest of the lines which are negatively temperaturedependent). As a result, the overall absorptivity effect is negative, indicating an enhanced trapping.

[19] It is worth noting that the water vapor continuum absorption is largely proportional to the square of water vapor density (especially in the window region, because of the self-broadening mechanism [Clough et al., 1989]). Thus the contribution by the continuum absorption is more sensitive to the water vapor concentration than the absorption lines. Because of the increase of water vapor concentration from midlatitudes to tropics (Table 1), the contribution by the continuum absorption compensates for the contribution by the lines to a larger extent at the tropical latitudes. As a result, the overall negative gas absorptivity effect becomes less compared to the Planck effect. This suggests that, in a warmer climate, assuming a uniform warming and consequent moistening of the atmosphere, the overall atmospheric temperature feedback (the "total OLR change" in Table 1) would be better approximated by the Planck effect alone.

\subsection{DLR}

[20] Similar to the analysis for OLR, it is apparent from Figure 5 that the Planck effect on DLR is offset by the absorptivity effect (the sum of terms (6b) and (6c)); this is mainly in the window region. Here, the absorptivity decreases with an increase in temperature. At the frequencies where line absorption dominates and is nearly saturated, the DLR sensitivity mostly results from the Planck effect. From Table 2, for the spectrally integrated DLR flux, if the

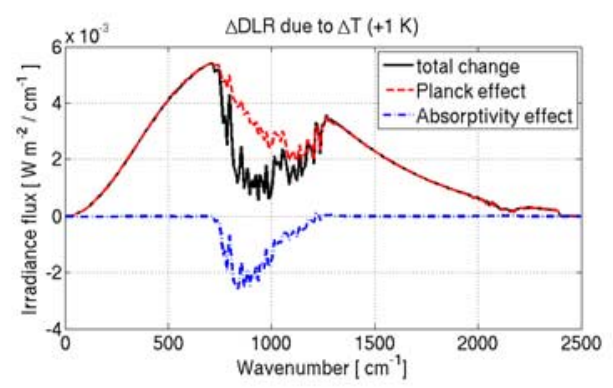

Figure 5. DLR $\left(\mathrm{W} \mathrm{m}^{-2} / \mathrm{cm}^{-1}\right)$ flux change due to $+1 \mathrm{~K}$ temperature perturbation, obtained from the spectral Jacobians (not shown). 
Table 2. Column Sum of the Irradiance Jacobians of DLR ${ }^{\mathrm{a}}$

\begin{tabular}{llll}
\hline & Tropics & MLS & MLW \\
\hline Total DLR change & 4.52 & 4.13 & 3.08 \\
Planck effect & 5.12 & 4.55 & 3.01 \\
Absorptivity effect & -0.59 & -0.42 & 0.07 \\
\hline
\end{tabular}

${ }^{a}$ Values are obtained as in Table 1.

whole tropical atmospheric column warms by $1 \mathrm{~K}$, the total absorptivity effect reduces the Planck effect $\left(5.12 \mathrm{~W} \mathrm{~m}^{-2}\right)$ by $-0.59 \mathrm{~W} \mathrm{~m}^{-2}$. In contrast, for the midlatitude winter case, the absorptivity effect is positive. This is because, as water vapor amount becomes less, the continuum absorption becomes negligible and the absorptivity effect arises almost entirely from the spectral regions with lines only. In going from tropics to higher latitudes, the temperature effect on DLR changes from sub-Planckian to super-Planckian.

[21] It is interesting to note that the total absorptivity effect tends to reduce both OLR and DLR (except in the midlatitude winter case). This feature indicates the nongray characteristic of the atmosphere. To demonstrate this point, consider the atmosphere to be a hypothetical single bulk layer. In a gray atmosphere, $O L R=\sigma T_{s}^{4}-\varepsilon\left(\sigma T_{s}^{4}-\sigma T_{a}^{4}\right)$ and $D L R=\varepsilon \sigma \mathrm{T}_{a}^{4}$, where $\varepsilon$ is the broadband emissivity of the atmosphere; $\sigma$ is the Stephan-Boltzman constant; $T_{a}$ is the effective atmospheric temperature; and $T_{s}$, the surface temperature. From these two equations, it is obvious that $\frac{\partial O L R}{\partial \varepsilon}<0$ while $\frac{\partial D L R}{\partial \varepsilon}>0$. Since, in fact, OLR and DLR changes are both negative (Tables 1 and 2), the characteristic feature is a consequence of the nongray atmosphere. This nongray feature results in a radiation energy convergence in the atmosphere; this would, in turn, reduce the total cooling of the atmosphere, compared to the situation when temperature dependence of the gas absorptivity is ignored. For OLR, the spectral regions with positively temperaturedependent absorption lines dominate, although this dominance decreases as the water vapor amount increases. However, for DLR, the negatively temperature-dependent continuum absorption in the window region determines the absorptivity effect in tropics and midlatitude summer where the water vapor is more abundant than in midlatitude winter.

[22] Although the total absorptivity effect on OLR is negative and is one of trapping, more radiation from the surface is allowed to leave the climate system (Table 1). This suggests that the temperature dependence of atmospheric absorption serves to damp the warming of the surface. On the other hand, the reduction of the downward radiation energy from the atmosphere to the surface (Table 2) helps to explain why the neglect of the temperature dependence of atmospheric absorption tends to give larger climate sensitivity, as shown by Augustsson and Ramanathan [1977].

\section{Conclusion and Discussion}

[23] By using radiative Jacobians, the temperature feedback can be separated into two distinct effects. One is the Planck effect which changes thermal emission according to Planck's Law. The other is the absorptivity effect which involves a change in gas absorptivity resulting from the temperature dependence.
[24] This study has quantitatively diagnosed the separate contributions from the components that constitute the gas absorptivity effect. It is demonstrated that these components are individually significant even though they largely cancel each other such that the Planck effect dominates the total OLR and DLR sensitivities.

[25] There are two significant cancellations with regard to the absorptivity effect. When a temperature change occurs within an atmospheric layer, the temperature dependence of gas absorption changes the emissivity of the perturbed layer. It also changes the transmission functions for radiation emanating from the layers below and the surface to the TOA, as well as the transmission functions for radiation emanating from the layers above to the surface. At a given frequency, the change of thermal emission at the perturbed layer is offset by the change in the transmission of the emission from the other layers and the surface. Further, because of the difference in the temperature dependence of gas absorptivity in different spectral intervals, the effect of the lines with positive temperature dependence is offset by (1) the rest of the lines and (2) the continuum absorption, both of whom have a negative temperature dependence.

[26] The OLR analyses reveal that, in the spectral regions of positive temperature dependence (e.g., those absorption lines off the band center), the absorptivity effect offsets the Planck damping which constitutes most of the negative temperature feedback. In the spectral regions of negative temperature dependence (e.g., water vapor continuum absorption), the temperature-dependent gas absorptivity effect adds to the Planck damping. It is interesting and very important to note that the negative temperature dependence of water vapor continuum absorption has a contrasting effect on DLR. When warming occurs at the surface and consequently in the atmosphere, this mechanism enables more emission from the surface to leave the atmospheresurface system and simultaneously reduces the downward radiation from the atmosphere to the surface. This would tend to lower the warming at the surface. Further, this mechanism is strongly sensitive to the concentration of lower tropospheric water vapor. With increased moisture in a warmer climate, the negative temperature dependence of the continuum absorption is likely to play a more prominent role. It will make the Planck effect more efficient in damping the warming of the atmosphere-surface system, and will also reduce the radiation emitted to the surface from a warmer atmosphere.

[27] The above results are inferred for clear sky conditions. Cloudy skies would add more degrees of freedom to the problem because they are relatively closer to black or grey spectral bodies, owing to the absence of strong spectral variation [Yamamoto et al., 1970]. Clouds may change the relative importance of continuum absorption and lines with respect to the temperature-dependent gas absorptivity effect. For example, an optically thick (blackbody-like) low cloud would limit the role of the water vapor continuum absorption by masking its temperature dependence, and thus make OLR sensitivity to temperature less Planck-effect-dominated; but DLR sensitivity more so. In contrast, an optically thin high cloud, if its absorption is less temperature-dependent than the lines, would limit the contributions from those lines that play an important role in the middle and upper 
troposphere. This would make the OLR sensitivity more determined by the Planck effect.

[28] Finally, the total temperature-dependent gas absorptivity effect tends to result in a radiation energy convergence that decreases the total cooling of the atmosphere. It is of interest for future works to investigate how the atmospheric cooling/heating profile is influenced by this effect.

[29] Acknowledgments. We thank Xianglei Huang and Dan Schwarzkopf for valuable comments. We also thank three anonymous reviewers who helped improve the quality of this paper.

\section{References}

Augustsson, T., and V. Ramanathan (1977), A radiative-convective model study of the $\mathrm{CO}_{2}$ climate problem, J. Atmos. Sci., 34, 448-451.

Clough, S. A., F. X. Kneizys, and R. W. Davies (1989), Line shape and the water vapor continuum, Atmos. Res., 23, 229-241.

Clough, S. A., M. J. Iacono, and J.-L. Moncet (1992), Line-by-line calculations of atmospheric fluxes and cooling rates: Application to water vapor, J. Geophys. Res., 97, 15,761-15,785.

Comier, J., J. Hodges, and J. Drummond (2005), Infrared water vapor continuum absorption at atmospheric temperatures, J. Chem. Phys., 122(11), 114-309.

Fels, S. B., and M. D. Schwarzkopf (1981), An efficient, accurate algorithm for calculating $\mathrm{CO}_{2} 15 \mu \mathrm{m}$ band cooling rates, J. Geophys. Res., 86(C2), $1205-1232$

Goody, R., and Y. Yung (1989), Radiation calculation in a clear atmosphere, in Atmospheric Radiation: Theoretical Basis, 2nd ed., pp. 216-287, Oxford Univ. Press, New York.

Huang, Y., V. Ramaswamy, and B. Soden (2007), An investigation of the sensitivity of the clear-sky outgoing longwave radiation to atmospheric temperature and water vapor, J. Geophys. Res., 112, D05104, doi:10.1029/ 2005JD006906.

Manabe, S., and R. Wetherald (1967), Thermal equilibrium of the atmosphere with a give distribution of relative humidity, J. Atmos. Sci., 3, $241-259$.

McClatchey, R. A., et al. (1972), Optical properties of the atmosphere, Environ. Res. Pap., 411, Air Force Cambridge Res. Lab., Hanscom Air Force Base, Mass.

Rothman, L. S., et al. (2003), The HITRAN molecular spectrospcopic database: Edition of 2000 including updates through 2001, J. Quant. Spectrosc. Radiat. Transfer, 82, 5-44.

Schwarzkopf, M. D., and V. Ramaswamy (1999), Radiative effects of $\mathrm{CH}_{4}$, $\mathrm{N}_{2} \mathrm{O}$, halocarbons and the foreign-broadened $\mathrm{H}_{2} \mathrm{O}$ continuum: A GCM experiment, J. Geophys. Res., 104(D8), 9467-9488.

Soden, B., and I. Held (2006), An assessment of climate feedbacks in coupled ocean-atmosphere models, J. Clim., 19(14), 3354-3360.

Stocker, T. F., et al. (2001), Physical climate processes and feedbacks, in Climate Change 2001: The Scientific Basis, pp. 417-470, Cambridge Univ. Press, New York.

Tobin, D. C., et al. (1999), Downwelling spectral radiance observations at the SHEBA ice station: Water vapor continuum measurements from 17 to 26 micron, J. Geophys. Res., 104, 2081-2092.

Yamamoto, G., et al. (1970), Radiative transfer in water clouds in the infrared region, J. Atmos. Sci., 27, 282-292.

Zhang, M. H., R. D. Cess, J. J. Hack, and J. T. Kiehl (1994), Diagnostic study of climate feedback processes in atmospheric GCMs, J. Geophys. Res., 99, 5525-5537.

Y. Huang, Program in Atmospheric and Oceanic Sciences, Princeton University, Princeton, NJ 08544, USA. (yi.huang@noaa.gov)

V. Ramaswamy, Geophysical Fluid Dynamics Laboratory, NOAA, Princeton, NJ 08540, USA. 